\title{
The effect of prohibiting outside food during COVID-19 pandemic on the body weight of schizophrenic patients taking olanzapine or clozapine: a retrospective self-controlled study
}

\author{
Chengfang Zhang ${ }^{1 \#}$, Xiaodan Wang ${ }^{2 \#}$, Chengping Hu${ }^{1 \#}$, Wenhua Ding ${ }^{3 \#}$, Han $\mathrm{Li}^{4,5 \#}$, Meng Wu ${ }^{6 \#}$, \\ Manji Hu ${ }^{1 \#}$, Qiang Wang ${ }^{1 \#}$, Xirong Sun ${ }^{1}$
}

${ }^{1}$ Shanghai Pudong New Area Mental Health Center, Tongji University School of Medicine, Shanghai, China; ${ }^{2}$ Department of Neurology \& Institute of Neurology, Rui Jin Hospital, Shanghai Jiao Tong University School of Medicine, Shanghai, China; ${ }^{3}$ The Affiliated Brain Hospital of Guangzhou Medical University, Guangzhou, China; ${ }^{4}$ Shanghai Mental Health Center, Shanghai Jiao Tong University, School of Medicine, Shanghai, China; ${ }^{5}$ Shanghai Key Laboratory of Psychotic Disorders, Shanghai, China; ${ }^{6}$ Shandong Provincial Key Laboratory of Radiation Oncology, Cancer Research Center, Shandong Cancer Hospital and Institute, Shandong First Medical University and Shandong Academy of Medical Sciences, Jinan, China Contributions: (I) Conception and design: C Zhang, M Wu; (II) Administrative support: C Hu, Q Wang, X Sun; (III) Provision of study materials or patients: H Li, W Ding (IV) Collection and assembly of data: X Wang; (V) Data analysis and interpretation: C Zhang, M Hu; (VI) Manuscript writing: All authors; (VII) Final approval of manuscript: All authors.

\#These authors contributed equally to this work.

Correspondence to: Chengfang Zhang. Shanghai Pudong New Area Mental Health Center, Tongji University School of Medicine, 165 Sanlin Road, Shanghai 200124, China. Email: callup1987@126.com; Xirong Sun. Shanghai Pudong New Area Mental Health Center, Tongji University School of Medicine, 165 Sanlin Road, Shanghai 200124, China. Email: sunxirong1974@163.com.

Backgrounda Olanzapine and clozapine are atypical antipsychotics (AAPs) with the greatest risk of weight gain, and changes in feeding behavior are among the most important underlying mechanisms. However, few studies have investigated the role of diet-alone interventions in improving individuals' weight gain by taking AAPs. In closed management mental hospitals of China, family members are allowed to bring food to patients regularly, causing patients to have caloric intake added to their 3 daily meals. However, during the global pandemic of coronavirus disease 2019 (COVID-19), bringing food to the hospital was temporarily prohibited in mental health institutions in China to prevent the spread of the virus. This study sought to compare the body weight and body mass index (BMI) changes of patients taking olanzapine or clozapine undergoing diet-alone interventions caused by this prohibition.

Methods: A retrospective self-controlled study was conducted on 90 patients with schizophrenia from a single-center treated with olanzapine or clozapine monotherapy, or combined with aripiprazole or ziprasidone which has a small metabolic impact. A paired-samples $t$-test was used to compare the changes in body weight and BMI before and after the 3-month prohibition, and general linear regression was used to analyze the effects of gender, age, disease course, duration of drug exposure, and equivalent dose on the BMI improvement. Also, the percentage of people who lost weight and that of individuals who lost $5 \%$ of their pre-prohibition body weight were calculated.

Results: Paired-samples $t$-test showed that after 3-month prohibition, the patients' body weight $(71.68 \pm 6.83$ vs. 66.91 $\pm 7.03, \mathrm{P}<0.001)$ and $\mathrm{BMI}(26.43 \pm 2.11$ vs. $24.63 \pm 1.81, \mathrm{P}<0.001)$ decreased significantly. Weight loss rate accounted for $99.1 \%$, and weight loss of $5 \%$ from the pre-prohibition body weight accounted for $71.8 \%$. General linear regression showed that the duration of drug exposure $(\beta=-0.678, \mathrm{P}<0.001)$ was significantly and negatively correlated with the BMI changes. No significant correlation of gender, age, disease course, or equivalent dose with BMI changes was found.

Conclusions: Diet-alone interventions facilitate weight loss in chronically hospitalized schizophrenia patients taking AAPs. Conduction of dietary intervention in the early stages of medication may yield greater benefits. 
Keywords! COVID-19 pandemic; caloric restriction; olanzapine; clozapine; weight

Submitted Dec 16, 2020. Accepted for publication Mar 17, 2021.

doi: $10.21037 /$ apm-20-2513

View this article at: http://dx.doi.org/10.21037/apm-20-2513

\section{Introduction}

Atypical antipsychotics (AAPs) have become first-line medications for the treatment of schizophrenia due to their superior overall efficacy and tolerability compared with conventional antipsychotics. Unfortunately, AAPs clinical utility is limited by the development of body weight gain in some individuals, contributing to the higher risk of type 2 diabetes and cardiovascular disease in schizophrenic people (1). Weight gain also increases the risk of recurrence because it is a significant detriment to medication compliance. It was reported that patients who are obese are 13 times more likely to discontinue medication than nonobese individuals (2). Randomized clinical trials have indicated that weight gain liability varies significantly across the range of AAPs. Clozapine and olanzapine medications are associated with the greatest risk of clinically significant weight gain, with other agents producing relatively lower risk levels. Risperidone and quetiapine generally show low to moderate levels of mean weight gain. Ziprasidone and aripiprazole treatment are generally associated with minimal mean weight gain (1).

The underlying mechanism of AAP-induced weight gain is far from elucidated and may involve changes in feeding behavior, reduced energy expenditure, and directly influence pathways of metabolic processes (3). Lifestyle interventions that include dietary changes, calorie restriction, and increased physical activity generally prove to be effective in losing weight (4). Weight gain in pair-fed olanzapine mice was similar to that in control mice when olanzapine-fed mice were restricted to the same amount of food consumed by those fed the control diet (5). However, few studies have explored whether diet-alone interventions could reduce the weight of individuals taking AAPs.

For long-term hospitalized patients in closed wards, food brought in by visitors is almost the only source of extra calories added to their 3 daily meals. However, during the global pandemic of coronavirus disease 2019 (COVID-19), bringing food into the hospital was temporarily prohibited in most mental health institutions of China to reduce infection risk. Against this background, a retrospective self-controlled study was conducted on 90 schizophrenic patients from 1 center who were treated with olanzapine or clozapine. Body weight and body mass index (BMI) before and after a 3-month prohibition was compared to examine whether diet-alone interventions could be beneficial for weight loss. This study was expected to evaluate the importance of dietary factors in AAP-induced weight gain and provide useful ideas for its clinical management.

\section{Methods}

\section{Ethics approval and consent}

The study was conducted in accordance with the Declaration of Helsinki (as revised in 2013). The study was approved by the Ethics Committee of Shanghai Pudong New Area Mental Health Center, Tongji University School of Medicine. All participants provided an informed consent agreement.

\section{Participants}

The raw data that support the findings from this study are available from the corresponding author upon reasonable request. This retrospective study included a series of weight data from schizophrenic patients who underwent olanzapine or clozapine medication in Shanghai Pudong New Area Mental Health Center, Tongji University School of Medicine. The inclusion criteria were as follows: (I) available data of patients hospitalized from January 27, 2020 to April 27, 2020, which was when the hospital prohibited visitors from bringing food into the psychiatric ward during the COVID-19 pandemic. (II) Meet the International Statistical Classification of Diseases and Related Health Problems, Tenth Revision (ICD10), criteria for schizophrenia. (III) Treated with olanzapine or clozapine monotherapy, or combined with aripiprazole or ziprasidone, which has a relatively small metabolic impact, and the antipsychotic treatment regimen had not been changed in the past 3 months. (IV) Meet Chinese standards for overweight $\left(24.0-27.9 \mathrm{~kg} / \mathrm{m}^{2}\right)$ or obesity $\left(\geq 28.0 \mathrm{~kg} / \mathrm{m}^{2}\right)$. (V) Patients with substance abuse, postmenopausal women 
using hormone replacement therapy, smoking, and patients using weight-loss agents or lipid-lowering agents were excluded. (VI) Patients with diabetes, hyperthyroidism, and cardiovascular disease were excluded. The enrolled patients had no serious diseases of the heart, liver, kidney, digestive tract, or other major organs.

\section{Study design and variables}

A retrospective self-controlled study was conducted. The change (before prohibition $v$ s. after prohibition) in BMI and body weight was set as the main outcome variable. The percentage of individuals who lost weight and the percentage of individuals who lost $5 \%$ (this percentage of weight loss is considered to reduce cardiovascular risk significantly) of their pre-prohibition body weight was also investigated. The correlation between BMI change and age, gender, course of the disease, equivalent dose, and drug exposure duration was analyzed. The dose of olanzapine or clozapine was converted into an equivalent dose according to the defined daily dose method (DDD method), with $1 \mathrm{mg}$ olanzapine being equivalent to $30 \mathrm{mg}$ clozapine (6).

\section{Statistical analysis}

All statistical analyses were performed using SPSS software version 20.0 (IBM-SPSS, Chicago, IL, USA). A Pairedsample $t$ test was used to assess the significance of the changes in body weight and BMI. General linear regression analysis was performed to determine the associations between changes of BMI and other factors, including gender, age, disease course, equivalent dose, and duration of drug exposure. Normally distributed quantitative data were expressed as mean \pm standard deviation (SD). Abnormally distributed quantitative data were expressed as [median (interquartile range)]. Qualitative data were expressed as frequency and percentage. Values of $\mathrm{P}<0.05$ were considered statistically significant. The percentage of individuals who lost weight and the percentage of those who lost $5 \%$ of their pre-prohibition body weight were calculated using Excel software (https://office.microsoft.com/excel).

\section{Results}

\section{Clinical characteristics}

A total of 90 patients were included in the study, including 39 males and 51 females. The median age of the population was 44 years. The median disease course of the population was 13 years. The median duration of drug exposure was 7.5 years. Olanzapine was taken as the main medication by 67 participants $(74.44 \%)$; among them, 52 were treated with olanzapine alone, 8 were treated with olanzapine and aripiprazole and 1 patient was treated with olanzapine and ziprasidone. A total of 23 patients $(25.56 \%)$ took clozapine as the main medication, including 19 treated with clozapine alone, 3 with clozapine and aripiprazole, and 1 with clozapine and ziprasidone. After converting clozapine into an equivalent dose of olanzapine, the median equivalent dose of this population was $15 \mathrm{mg}$. A total of 73 cases were overweight $(81.11 \%)$, and 17 participants reached the standards of obesity (18.89\%). The mean weight of this population was $71.68 \pm 6.83 \mathrm{~kg}$, and the median BMI was $25.91 \mathrm{~kg} / \mathrm{m}^{2}$. Participant baseline characteristics are summarized in Table 1.

\section{Changes in body weight and BMI before and after the probibition and correlation analysis}

Paired-sample $t$-test showed that after 3 -month prohibition, the participants' body weight $(71.68 \pm 6.83$ vs. $66.91 \pm 7.03$, $\mathrm{P}<0.001)$ and $\mathrm{BMI}(26.43 \pm 2.11$ vs. $24.63 \pm 1.81, \mathrm{P}<0.001)$ decreased significantly (Table 2). Weight loss rate accounted for $99.1 \%$, and $5 \%$ weight loss of the pre-prohibition body weight accounted for $71.8 \%$. General linear regression showed that the duration of drug exposure $(\beta=-0.678$, $\mathrm{P}<0.001)$ was significantly and negatively correlated with the BMI changes. There was no significant correlation found between gender, age, disease course, equivalent dose and BMI changes (Table 3).

\section{Discussion}

Diet is a major and modifiable cause of cardiovascular disease, as a poor diet may be strongly linked to insulin resistance, dyslipidemia and hypertension (7). Only 1 study has explored diet-alone interventions instead of comprehensive interventions on the effect of weight change in short-term acute care psychiatric patients receiving AAPs, which is effective for patients taking olanzapine (8). However, whether diet-alone interventions could reduce the weight of chronic closed ward hospitalized schizophrenia patients who take AAPs has not yet been reported. Compared to outpatients or acute patients, metabolic studies conducted among chronically hospitalized populations may have fewer confounding factors, which has 
Table 1 Descriptive statistics

\begin{tabular}{lc}
\hline Index & Outcome (N=90) \\
\hline Gender (male/female) & $39 / 51$ \\
Age [median (interquartile range)] (years) & $44.00(33.00-50.50)$ \\
AAPs & $67(74.44)$ \\
Olanzapine [n (\%)] & $23(25.56)$ \\
Clozapine [n (\%)] & $13[8-17]$ \\
Disease course [median (interquartile range)] (years) & $15.00(10.00-20.00)$ \\
Equivalent dose [median (interquartile range)] (mg) & $7.5[4-9]$ \\
Duration of drug exposure [median (interquartile range)] (years) & $25.91(24.80-27.24)$ \\
Pre-BMI [median (interquartile range)] (kg/m ${ }^{2}$ ) & $73(81.11)$ \\
Overweight [n (\%)] & $17(18.89)$ \\
Obesity [n (\%)] & 71.68 (6.83) \\
Pre-weight [mean (SD)] (kg) &
\end{tabular}

AAPs, atypical antipsychotics; Pre-BMI, pre-prohibition body mass index; Pre-weight, pre-prohibition body weight.

Table 2 Changes in body weight and BMI before and after the prohibition $(\mathrm{N}=90)$

\begin{tabular}{lcc}
\hline & Body weight $(\mathrm{kg})$ & $\mathrm{BIM}\left(\mathrm{kg} / \mathrm{m}^{2}\right)$ \\
\hline Pre-prohibition (mean $\pm \mathrm{SD})$ & $71.68 \pm 6.83$ & $26.43 \pm 2.11$ \\
Post-prohibition (mean $\pm \mathrm{SD})$ & $66.91 \pm 7.03$ & $24.63 \pm 1.81$ \\
$\mathrm{t}$ & 18.25 & 16.81 \\
$\mathrm{P}$ & $<0.001$ & $<0.001$ \\
\hline
\end{tabular}

BMI, body mass index

been reflected in greater consistency in their activity levels and dietary structure delivered by shared living space.

A before-after study was applied to retrospectively explore the body weight and BMI of chronic schizophrenia patients taking olanzapine or clozapine after a 3-month prohibition of bringing food into the psychiatric ward to minimize transmission of COVID-19. After 3 months of restricting any caloric intake in addition to their 3 daily meals, the body weight and BMI of these patients improved significantly, suggesting that increased caloric intake plays an important role in weight gain associated with olanzapine or clozapine, and dietary intervention is a powerful approach of weight management. A weight loss of $5 \%$ is considered clinically important for health, especially in reducing the risk of cardiovascular diseases (9). In our study, up to $71.8 \%$ of participants lost $5 \%$ of their pre-prohibition body weight, suggesting that diet-alone interventions may have cardiovascular benefits for most patients participating in the study. However, this seems to be higher than other studies' conclusions, including STRIDE that lifestyle interventions showed clinically significant weight loss of $\geq 5 \%$ of initial body weight among $40 \%$ of participants (10). We speculate that the reason for our more promising results may be that all of our included participants were taking olanzapine or clozapine, rather than other AAPs, which are considered to have the most serious effects on increased food intake (11), leading to a more significant effect of calorie restriction on weight gain, as suggested by the results of Jacobowitz et al. (8).

We explored the factors that may affect weight loss brought by this "passive" diet intervention, including gender, age, disease course, equivalent dose, and drug exposure duration. Our data suggested that improvement in weight is negatively correlated with the duration of drug exposure. Although there are few studies on the correlation between the effects of lifestyle intervention and the duration of AAP exposure, it has been suggested that metabolic syndrome is associated with a longer duration of psychiatric treatment (12). Moreover, in the early stage of using olanzapine, the increase of appetite is more obvious, weight gain is more rapider, and the early increase of appetite predicts a longterm weight gain (13). The only randomized controlled 
Table 3 General linear regression analysis of age, gender, and other factors associated with changes of BMI

\begin{tabular}{|c|c|c|c|c|c|}
\hline Index & Unstandardized B & Coefficients Std. error & Standardized coefficients $\beta$ & $\mathrm{t}$ & Sig. \\
\hline Gender & -0.189 & 0.193 & -0.093 & -0.979 & 0.33 \\
\hline Age & 0.019 & 0.01 & 0.175 & 1.994 & 0.05 \\
\hline Disease course & 0.003 & 0.011 & 0.017 & 0.223 & 0.82 \\
\hline Duration of drug exposure & -0.185 & 0.024 & -0.678 & -7.618 & $<0.001$ \\
\hline
\end{tabular}

BMI, body mass index

trial (RCT) that evaluated an early intervention in young patients with recent-onset psychosis showed a significantly attenuated weight gain (14). Therefore, metabolic benefits may be greater if calorie restriction are carried out from when the antipsychotic treatment is initiated. The treatment response of schizophrenia in the initial stage of drug treatment is better, and it is more important for determining the prognosis of the disease. Subsequently, early weight-management interventions have the potential to be more cost-efficient and beneficial. We did not find a significant correlation between gender, age, course of disease, or equivalent dose with weight improvement, which is consistent with previous studies' conclusions that the effects of these factors on weight improvement brought by life-style interventions are not yet clear $(8,15)$.

This research may suggest that early dietary intervention is very important for patients taking AAPs who are at risk of causing weight gain. However, for outpatients, the implementation of dietary interventions is undoubtedly full of challenges. Because of negative symptoms such as amotivation and apathy, lifestyle interventions, including diet control among schizophrenic individuals, often require increased social resources and higher economic costs to improve participation and adherence (16). Moreover, changes in feeding behaviors caused by AAPs may involve changes in satiety perception, increases in appetite and food intake, and poor dietary choices, increasing the difficulty of dietary intervention (3). Therefore, finding the pharmacological targets by exploring the mechanisms underlying the feeding behavior changes and developing safe drugs may be the fundamental way to solve AAPinduced weight gain.

\section{Conclusions}

Diet-alone interventions may be efficient in reducing AAP-associated weight gain in chronically hospitalized schizophrenia patients. The effect of dietary interventions is negatively correlated with the duration of drug exposure, suggesting that conducting dietary interventions from the time of antipsychotic treatment initiation may have the potential to be more beneficial.

\section{Limitations}

Firstly, given this research is a retrospective study, the participants' daily calorie intake was not quantified, even if all patients received uniform meals from the hospital. Secondly, this study lacks the data of baseline weight of patients before initiating the use of AAPs. Thirdly, the participants' psychiatric symptoms and daily activity level have not been quantitatively assessed. Therefore, further studies and long-term follow-up are warranted in the future.

\section{Acknowledgments}

We would like to thank J. Jones and J. Chapnick for their help in polishing our paper.

Funding: Science and Technology Development Fund of Shanghai Pudong New Area (PKJ2018-Y27). Excellent Youth Medical Talents Training Program of Pudong Health Bureau of Shanghai (PWRq2020-19). Key Discipline Construction Project of Pudong Health Bureau of Shanghai (PWZxk2017-29). The Outstanding Clinical Discipline Project of Shanghai Pudong (PWYgy2018-10). China Postdoctoral Science Foundation (2018M640769). 


\section{Footnote}

Provenance and Peer Review: This article was commissioned by the editorial office, Annals of Palliative Medicine for the series "International Conference on Palliative Care and Medicine". The article has undergone external peer review.

Conflicts of Interest: All authors have completed the ICMJE uniform disclosure form (available at http://dx.doi. org/10.21037/apm-20-2513). The series "International Conference on Palliative Care and Medicine" was commissioned by the editorial office without any funding or sponsorship. The authors have no other conflicts of interest to declare.

Ethical Statement: The authors are accountable for all aspects of the work in ensuring that questions related to the accuracy or integrity of any part of the work are appropriately investigated and resolved. The study was conducted in accordance with the Declaration of Helsinki (as revised in 2013). The study was approved by the Ethics Committee of Shanghai Pudong New Area Mental Health Center, Tongji University School of Medicine. All participants provided an informed consent agreement.

Open Access Statement: This is an Open Access article distributed in accordance with the Creative Commons Attribution-NonCommercial-NoDerivs 4.0 International License (CC BY-NC-ND 4.0), which permits the noncommercial replication and distribution of the article with the strict proviso that no changes or edits are made and the original work is properly cited (including links to both the formal publication through the relevant DOI and the license). See: https://creativecommons.org/licenses/by-nc-nd/4.0/.

\section{References}

1. Newcomer JW. Second-generation (atypical) antipsychotics and metabolic effects: a comprehensive literature review. CNS Drugs 2005;19 Suppl 1:1-93.

2. Weiden PJ, Mackell JA, McDonnell DD. Obesity as a risk factor for antipsychotic noncompliance. Schizophr Res 2004;66:51-7.

3. Benarroch L, Kowalchuk C, Wilson V, et al. Atypical antipsychotics and effects on feeding: from mice to men. Psychopharmacology 2016;233:2629-53.

4. Bonfioli E, Berti L, Goss C, et al. Health promotion lifestyle interventions for weight management in psychosis: a systematic review and meta-analysis of randomised controlled trials. BMC Psychiatry 2012;12:78.

5. Lord CC, Wyler SC, Wan R, et al. The atypical antipsychotic olanzapine causes weight gain by targeting serotonin receptor 2C. J Clin Invest 2017;127:3402-6.

6. Leucht S, Samara M, Heres S, et al. Dose Equivalents for Antipsychotic Drugs: The DDD Method. Schizophr Bull 2016;42 Suppl 1:S90-4.

7. Siri-Tarino PW, Sun Q, Hu FB, et al. Saturated fat, carbohydrate, and cardiovascular disease. Am J Clin Nutr 2010;91:502-9.

8. Jacobowitz W, Derbabian B, Saunders A. The effect of a calorie-restricted diet on weight gain in short-term psychiatric inpatients receiving atypical antipsychotic medications. J Psychosoc Nurs Ment Health Serv 2014;52:30-7.

9. Moyer VA. Screening for and management of obesity in adults: U.S. Preventive Services Task Force recommendation statement. Ann Intern Med 2012;157:373-8.

10. Green CA, Yarborough BJ, Leo MC, et al. The STRIDE weight loss and lifestyle intervention for individuals taking antipsychotic medications: a randomized trial. Am J Psychiatry 2015;172:71-81.

11. Cuerda C, Velasco C, Merchán-Naranjo J, et al. The effects of second-generation antipsychotics on food intake, resting energy expenditure and physical activity. Eur J Clin Nutr 2014;68:146-52.

12. Misawa F, Shimizu K, Fujii Y, et al. Is antipsychotic polypharmacy associated with metabolic syndrome even after adjustment for lifestyle effects?: a cross-sectional study. BMC Psychiatry 2011;11:118.

13. Kinon BJ, Kaiser CJ, Ahmed S, et al. Association between early and rapid weight gain and change in weight over one year of olanzapine therapy in patients with schizophrenia and related disorders. J Clin Psychopharmacol 2005;25:255-8.

14. Alvarez-Jiménez M, Hetrick SE, González-Blanch C, et al. Non-pharmacological management of antipsychoticinduced weight gain: systematic review and metaanalysis of randomised controlled trials. Br J Psychiatry 2008;193:101-7.

15. Marteene W, Winckel K, Hollingworth S, et al. Strategies to counter antipsychotic-associated weight gain in patients with schizophrenia. Expert Opin Drug 
Saf 2019;18:1149-60.

16. Meenan RT, Stumbo SP, Yarborough MT, et al. An Economic Evaluation of a Weight Loss Intervention
Program for People with Serious Mental Illnesses Taking Antipsychotic Medications. Adm Policy Ment Health 2016;43:604-15.

Cite this article as: Zhang C, Wang $\mathrm{X}, \mathrm{Hu} \mathrm{C}$, Ding W, Li H, Wu M, Hu M, Wang Q, Sun X. The effect of prohibiting outside food during COVID-19 pandemic on the body weight of schizophrenic patients taking olanzapine or clozapine: a retrospective self-controlled study. Ann Palliat Med 2021;10(5):5010-5016. doi: 10.21037/apm-20-2513 